\title{
Large Eddy Simulation-Based Analysis of Entropy Generation in a Turbulent Nonpremixed Flame
}

\author{
Mehdi Safari Qariq \\ Fairfield University, msafariqariq@fairfield.edu \\ M. Reza H. Sheikhi
}

Follow this and additional works at: https://digitalcommons.fairfield.edu/engineering-facultypubs Copyright (c) 2014 Elsevier Ltd. All rights reserved.

The author post-print has been archived here with permission from the copyright holder.

\section{Peer Reviewed}

\section{Repository Citation}

Safari Qariq, Mehdi and H. Sheikhi, M. Reza, "Large Eddy Simulation-Based Analysis of Entropy Generation in a Turbulent Nonpremixed Flame" (2014). Engineering Faculty Publications. 131.

https://digitalcommons.fairfield.edu/engineering-facultypubs/131

\section{Published Citation}

Safari, M. and Sheikhi, M.R.H. "Large Eddy Simulation-Based Analysis of Entropy Generation in a Turbulent Nonpremixed Flame," Energy, Vol. 78, 2014, pp.451-457.

This item has been accepted for inclusion in DigitalCommons@Fairfield by an authorized administrator of DigitalCommons@Fairfield. It is brought to you by DigitalCommons@Fairfield with permission from the rightsholder(s) and is protected by copyright and/or related rights. You are free to use this item in any way that is permitted by the copyright and related rights legislation that applies to your use. For other uses, you need to obtain permission from the rights-holder(s) directly, unless additional rights are indicated by a Creative Commons license in the record and/or on the work itself. For more information, please contact digitalcommons@fairfield.edu. 


\title{
Large Eddy Simulation-Based Analysis of Entropy Generation in a Turbulent Nonpremixed Flame
}

\author{
M. Safari ${ }^{\text {a }}$ and M.R.H. Sheikhi ${ }^{\text {a,* }}$

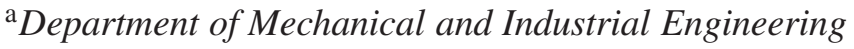 \\ Northeastern University \\ Boston, MA 02115
}

\begin{abstract}
Large eddy simulation (LES) is employed for prediction and analysis of entropy generation in turbulent combustion. The entropy transport equation is considered in LES. This equation contains unclosed entropy generation terms due to irreversible processes: heat conduction, mass diffusion, chemical reaction and viscous dissipation. The subgrid scale (SGS) closure of these terms is provided by a methodology termed the entropy filtered density function (En-FDF), which contains complete statistical information about SGS variation of scalars and entropy. In the En-FDF, the effects of chemical reaction and its entropy generation appear in closed forms. This methodology is used for LES of a nonpremixed jet flame. Predictions show good agreements with the experimental data. Analysis of entropy generation shows that heat conduction and chemical reaction are the main sources of irreversibility in this flame. The sensitivity of individual entropy generation effects to turbulence intensity is studied.
\end{abstract}

\footnotetext{
* Corresponding author: E-mail: sheikhi@ neu.edu, Tel.: 617-373-3427.
} 


\section{Introduction}

Improved energy efficiency is a key objective in development of modern energy systems. To achieve optimum efficiency in energy conversion it is essential to minimize the irreversible losses in the system. In practice, transport processes are always accompanied by irreversible losses, causing destruction of exergy (availability) of the working-fluid and thus decreased energy efficiency, from the second law of thermodynamics standpoint [1]. The rate of exergy destruction due to irreversibilities is characterized in terms of entropy generation according to the Gouy-Stodola theorem, $I_{D}=T_{0} S_{g}$ [2,3], where $I_{D}, T_{0}$ and $S_{g}$ denote the rate of exergy destruction (also known as lost power), ambient (dead state) temperature and entropy generation rate, respectively. Therefore, increasing the energy efficiency relies on reducing the overall exergy destruction which depends on minimization of entropy generated within the system [4-9]. During the past several decades the second-law analysis has been the subject of broad investigations. A system-level analysis, often termed exergy analysis, is used to obtain the net rate of exergy destruction [10-23]. Alternatively, analysis of local generation of entropy reveals the specific agents contributing to irreversible losses. Such analysis applied to laminar flows has been the subject of many studies. Teng et al. [24] derived the entropy transport equation to determine the rate of local entropy generation in multicomponent laminar reacting flows. Datta \& Som [18] considered energy and exergy balance in a gas turbine combustor. Datta [25] conducted entropy generation analysis of a laminar diffusion flame. Nishida et al. [26] considered premixed and diffusion flames and identified important entropy generation and exergy loss mechanisms. Datta [27] studied the effect of gravity on structure and generation of entropy in confined laminar diffusion flames. Shuja et al. [28] studied the influence of inlet velocity profile on efficiency of heat transfer in a laminar jet. 
Briones et al. [29] studied the entropy generation processes in a partially-premixed flame. Sciacovelli \& Verda [30] used entropy generation minimization technique for design modifications in a tubular solid oxide fuel cell. Jiang et al. [31] presented an analysis of entropy generation in a hydrogen/air premixed micro-combustor with baffles and Rana et al. [32] studied the exergy transfer and destruction due to premixed combustion in a heat recirculating micro-combustor.

In turbulent flows, there has been several studies on entropy generation via direct numerical simulation (DNS). Okong'o \& Bellan [33-35] performed comprehensive studies on entropy generation effects in supercritical, multicomponent shear flows; they suggested that, by containing the full extent of dissipative effects, entropy generation is useful to describe the behavior of small-scale turbulent motions. McEligot et al. [36] studied the entropy generation in the near wall region of a turbulent channel flow. Farran \& Chakraborty [37] conducted DNS prediction of entropy generation in a turbulent premixed flame. Simulations based on modeled entropy generation effects have mainly been in the context of Reynolds Averaged Navier-Stokes (RANS) simulations. Stanciu et al. [38] performed the second-law analysis of a turbulent diffusion flame. Shuja et al. [39] studied local entropy generation in an impinging jet and used minimum entropy concept to evaluate various turbulence models. Adeyinka \& Naterer [40] provided a model for entropy transport equation in turbulent flows. Kock \& Herwig [41] provided wall functions for entropy production and performed analysis of entropy generation due to fluid flow and heat transfer in near wall region of a pipe. Yapici et al. [42] performed local entropy generation in a methane-air burner. Herwig \& Kock [43] used entropy generation as a tool for evaluating heat transfer performance in a turbulent shear flow. Stanciu et al. [44] studied the influence of swirl 
angle on the irreversibility in a turbulent diffusion flame and Emadi \& Emami [45] studied entropy generation in a turbulent hydrogen enriched methane/air bluff-body flame. Despite the benefits of large eddy simulation (LES) in turbulence modeling, its application for entropy generation analysis has been very limited because of challenges to subgrid scale (SGS) modeling of the unclosed irreversibility effects. An effective strategy for modeling of SGS effects is the filtered density function (FDF) methodology $[46,47]$. This methodology has been the subject of extensive previous contributions [48-60]. In recent works [61,62], a FDF-based methodology, termed the entropy FDF (En-FDF) has been introduced which allows LES prediction of entropy transport and generation in turbulent reacting flows. In this study, the marginal form of this methodology [62] is considered which contains the complete statistical information on entropy and scalar fields and thus, accounts for individual entropy generation effects in turbulent reacting flows. The En-FDF is applied to predict a nonpremixed methane jet flame (Sandia Flame D) [63]. The objectives of the present study are to assess the accuracy of the En-FDF and to demonstrate its effectiveness for prediction and analysis of entropy generation in turbulent reacting flows.

\section{Mathematical modeling}

We consider the compressible form of the continuity, Navier-Stokes, energy (enthalpy), mass fraction and entropy transport equations in low Mach number flows. Along with the ideal gas equation of state, these equations describe transport of fluid density $\rho(\mathbf{x}, t)$, the velocity vector $u_{i}(\mathbf{x}, t)$, the pressure $p(\mathbf{x}, t)$, the specific enthalpy $h(\mathbf{x}, t)$, mass fraction of species $Y_{\alpha}(\mathbf{x}, t)\left(\alpha=1, \ldots, N_{s}\right)$ and the specific entropy $s(\mathbf{x}, t)$. Large eddy simulation involves the use of filtering operation

$$
\langle Q(\mathbf{x}, t)\rangle=\int_{-\infty}^{+\infty} Q\left(\mathbf{x}^{\prime}, t\right) G\left(\mathbf{x}^{\prime}, \mathbf{x}\right) d \mathbf{x}^{\prime}
$$


where $\langle Q\rangle$ is the filtered variable and $G$ is the filter function with characteristic width $\Delta$. We consider the filter functions which is spatially invariant, localized and symmetric with $\int_{-\infty}^{+\infty} G(\mathbf{x}) d \mathbf{x}=1$. We assume existence of all moments $\int_{-\infty}^{+\infty} \mathbf{x}^{m} G(\mathbf{x}) d \mathbf{x}$ $(m \geq 0)$. In compressible flows we use the Favre filter variable $\langle Q\rangle_{L}=\langle\rho Q\rangle /\langle\rho\rangle$. Applying the filtering operation to the transport equations we obtain

$$
\begin{gathered}
\frac{\partial\langle\rho\rangle}{\partial t}+\frac{\partial\langle\rho\rangle\left\langle u_{i}\right\rangle_{L}}{\partial x_{i}}=0 \\
\frac{\partial\langle\rho\rangle\left\langle u_{i}\right\rangle_{L}}{\partial t}+\frac{\partial\langle\rho\rangle\left\langle u_{i}\right\rangle_{L}\left\langle u_{j}\right\rangle_{L}}{\partial x_{j}}=-\frac{\partial\langle p\rangle}{\partial x_{i}}+\frac{\partial\left\langle\tau_{i j}\right\rangle_{L}}{\partial x_{j}}-\frac{\partial\langle\rho\rangle \tau\left(u_{i}, u_{j}\right)}{\partial x_{j}} \\
\frac{\partial\langle\rho\rangle\left\langle\phi_{\alpha}\right\rangle_{L}}{\partial t}+\frac{\partial\langle\rho\rangle\left\langle u_{i}\right\rangle_{L}\left\langle\phi_{\alpha}\right\rangle_{L}}{\partial x_{i}}=\frac{\partial}{\partial x_{i}}\left(\gamma \frac{\partial\left\langle\phi_{\alpha}\right\rangle_{L}}{\partial x_{i}}\right)-\frac{\partial\langle\rho\rangle \tau\left(u_{i}, \phi_{\alpha}\right)}{\partial x_{i}}+\left\langle\rho S_{\alpha}\right\rangle \\
\frac{\partial\langle\rho\rangle\langle s\rangle_{L}}{\partial t}+\frac{\partial\langle\rho\rangle\left\langle u_{i}\right\rangle_{L}\langle s\rangle_{L}}{\partial x_{i}}=\frac{\partial}{\partial x_{i}}\left(\gamma \frac{\partial\langle s\rangle_{L}}{\partial x_{i}}\right)-\frac{\partial\langle\rho\rangle \tau\left(u_{i}, s\right)}{\partial x_{i}} \\
+\left\langle\frac{1}{T} \tau_{i j} \frac{\partial u_{i}}{\partial x_{j}}\right\rangle+\left\langle\gamma \frac{c_{p}}{T^{2}} \frac{\partial T}{\partial x_{i}} \frac{\partial T}{\partial x_{i}}\right\rangle+\sum_{\alpha=1}^{N_{s}}\left\langle\frac{\gamma R_{\alpha}}{X_{\alpha}} \frac{\partial \phi_{\alpha}}{\partial x_{i}} \frac{\partial X_{\alpha}}{\partial x_{i}}\right\rangle-\left\langle\frac{\rho}{T} \sum_{\alpha=1}^{N_{s}} \mu_{\alpha} S_{\alpha}\right\rangle
\end{gathered}
$$

where $R_{\alpha}, X_{\alpha}, \mu_{\alpha}$ and $S_{\alpha}$ are gas constant, mole fraction, specific chemical potential and chemical reaction source term for species $\alpha$, respectively. Variables $T$ and $c_{p}$ denote the temperature and the specific heat capacity at constant pressure for the mixture; $\gamma$ denotes the thermal and mass molecular diffusivity coefficients for all the scalars. We assume unity Lewis number and we set the molecular Schmidt (and Prandtl) number as $S c=\operatorname{Pr}=0.75$. We use the scalar array $\phi=\left[\phi_{1}, \ldots, \phi_{N_{s}+1}\right]$ to represent mass fraction and enthalpy in a common form with $\phi_{\alpha} \equiv Y_{\alpha}$ for $\alpha=1, \ldots, N_{s}$ and $\phi_{N_{s}+1} \equiv h$. In these equations, we employ Fourier's law of heat conduction and Fick's law of diffusion and we assume a Newtonian fluid with the molecular stress tensor $\tau_{i j}=\mu\left(\frac{\partial u_{i}}{\partial x_{j}}+\frac{\partial u_{j}}{\partial x_{i}}-\frac{2}{3} \frac{\partial u_{k}}{\partial x_{k}} \delta_{i j}\right)$ where $\mu$ denotes the molecular viscosity which is proportional to $T^{0.7}$. Equation (5) is the filtered entropy transport equation [64]. The irreversible generation of entropy in this equation is described by the last four terms 
which correspond to viscous dissipation $S_{g_{V}}$, heat conduction $S_{g_{H}}$, mass diffusion $S_{g_{M}}$ and chemical reaction $S_{g_{C}}$, respectively. According to the second law of thermodynamics, the modeled filtered entropy generation terms must be positive semidefinite. The closure problem in Eqs. (2-5) is associated with unclosed SGS entropy flux terms $\tau\left(u_{i}, s\right)$, where $\tau(a, b)=\langle a b\rangle_{L}-\langle a\rangle_{L}\langle b\rangle_{L}$. In addition, the filtered chemical reaction source term (the last term on the RHS of Eq. (4)) and the entropy generation terms (the last four term on the RHS of Eq. (5)) appear in unclosed forms. For modeling of SGS stress tensor we employ the Modified Kinetic Energy Viscosity (MKEV) closure [49]. To represent the scalar and entropy flux terms we use $\gamma_{t}=v_{t} / S c_{t}$, where the SGS viscosity $v_{t}$ is described by MKEV and the turbulent Schmidt (and Prandtl) number is $S c_{t}=0.75$.

The closure of chemical reaction source term and the entropy generation effects is provided by the En-FDF methodology, denoted by $\mathscr{F}_{\text {en }}(\hat{\boldsymbol{\phi}}, \hat{s}, \mathbf{x} ; t)$. The En-FDF contains complete statistical information about scalar and entropy fields and is formally defined as

$$
\mathscr{F}_{e n}(\hat{\boldsymbol{\phi}}, \hat{s}, \mathbf{x} ; t)=\int_{-\infty}^{+\infty} \rho\left(\mathbf{x}^{\prime}, t\right) \times \zeta\left[\hat{\boldsymbol{\phi}}, \hat{s} ; \boldsymbol{\phi}\left(\mathbf{x}^{\prime}, t\right), s\left(\mathbf{x}^{\prime}, t\right)\right] G\left(\mathbf{x}^{\prime}-\mathbf{x}\right) d \mathbf{x}^{\prime}
$$

where

$$
\zeta[\hat{\boldsymbol{\phi}}, \hat{s} ; \boldsymbol{\phi}(\mathbf{x}, t), s(\mathbf{x}, t)]=\delta(\hat{s}-s(\mathbf{x}, t)) \times \prod_{\alpha=1}^{N_{s}+1} \delta\left(\hat{\phi}_{\alpha}-\phi_{\alpha}(\mathbf{x}, t)\right)
$$

is the fine-grained density [65]; $\delta$ denotes the Dirac delta function. The sample space variables $\hat{\boldsymbol{\phi}}$ and $\hat{s}$ correspond to scalar array and entropy, respectively. The marginal 
En-FDF is governed by an exact transport equation

$$
\begin{aligned}
& \frac{\partial \mathscr{F}_{e n}}{\partial t}+\frac{\partial\left[\left\langle u_{i} \mid \hat{\boldsymbol{\phi}}, \hat{s}\right\rangle \mathscr{F}_{e n}\right]}{\partial x_{i}}=-\sum_{\alpha=1}^{N_{s}+1} \frac{\partial}{\partial \hat{\phi}_{\alpha}}\left[\left\langle\frac{1}{\rho} \frac{\partial}{\partial x_{i}}\left(\gamma \frac{\partial \phi_{\alpha}}{\partial x_{i}}\right) \mid \hat{\boldsymbol{\phi}}, \hat{s}\right\rangle \mathscr{F}_{e n}\right] \\
& -\frac{\partial}{\partial \hat{s}}\left[\left\langle\frac{1}{\rho} \frac{\partial}{\partial x_{i}}\left(\gamma \frac{\partial s}{\partial x_{i}}\right) \mid \hat{\boldsymbol{\phi}}, \hat{s}\right\rangle \mathscr{F}_{e n}\right]-\frac{\partial}{\partial \hat{s}}\left[\left\langle\gamma \frac{c_{p}}{\rho T^{2}} \frac{\partial T}{\partial x_{i}} \frac{\partial T}{\partial x_{i}} \mid \hat{\boldsymbol{\phi}}, \hat{s}\right\rangle \mathscr{F}_{e n}\right] \\
& -\frac{\partial}{\partial \hat{s}}\left[\left\langle\frac{\gamma}{\rho} \sum_{\alpha=1}^{N_{s}} \frac{R_{\alpha}}{X_{\alpha}} \frac{\partial \phi_{\alpha}}{\partial x_{i}} \frac{\partial X_{\alpha}}{\partial x_{i}} \mid \hat{\boldsymbol{\phi}}, \hat{s}\right\rangle \mathscr{F}_{e n}\right]-\frac{\partial}{\partial \hat{s}}\left[\left\langle\frac{1}{\rho T} \tau_{i j} \frac{\partial u_{i}}{\partial x_{j}} \mid \hat{\boldsymbol{\phi}}, \hat{s}\right\rangle \mathscr{F}_{e n}\right] \\
& -\sum_{\alpha=1}^{N_{s}} \frac{\partial}{\partial \hat{\phi}_{\alpha}}\left[S_{\alpha}(\hat{\boldsymbol{\phi}}) \mathscr{F}_{e n}\right]+\frac{\partial}{\partial \hat{s}}\left[\frac{1}{T} \sum_{\alpha=1}^{N_{s}} \mu_{\alpha} S_{\alpha}(\hat{\boldsymbol{\phi}}) \mathscr{F}_{e n}\right]
\end{aligned}
$$

where $\langle\mid\rangle$ denotes the conditional filtered values [54]. In this equation, the effects of chemical reaction (the last two terms on the RHS) are in closed forms. However, all the terms involving conditional filtered values require closure. The closure of the EnFDF is provided by a set of stochastic differential equations (SDEs) corresponding to position, scalars and entropy [62]

$$
\begin{aligned}
d X_{i}^{+} & =\left(\left\langle u_{i}\right\rangle_{L}+\frac{1}{\langle\rho\rangle} \frac{\partial\left(\gamma+\gamma_{t}\right)}{\partial x_{i}}\right) d t+\left(\sqrt{\frac{2\left(\gamma+\gamma_{t}\right)}{\langle\rho\rangle}}\right) d W_{i} \\
d \phi_{\alpha}^{+} & =-C_{\phi} \Omega\left(\phi_{\alpha}^{+}-\left\langle\phi_{\alpha}\right\rangle_{L}\right) d t+S_{\alpha}\left(\phi^{+}\right) d t \\
d s^{+} & =\frac{\epsilon_{t}}{T^{+}} d t+\frac{1}{T^{+}} \sum_{\alpha=1}^{N_{s}} \mu_{\alpha}^{+} C_{\phi} \Omega\left(\phi_{\alpha}^{+}-\left\langle\phi_{\alpha}\right\rangle_{L}\right) d t \\
& -\frac{1}{T^{+}} C_{\phi} \Omega\left(h^{+}-\langle h\rangle_{L}\right) d t-\frac{1}{T^{+}} \sum_{\alpha=1}^{N_{s}} \mu_{\alpha}^{+} S_{\alpha}\left(\phi^{+}\right) d t
\end{aligned}
$$

where $X_{i}^{+}, \phi_{\alpha}^{+}, T^{+}, \mu_{\alpha}^{+}$and $s^{+}$are the stochastic representations of position, scalars, temperature, chemical potential per unit mass of species $\alpha$ and entropy, respectively. The variable $W_{i}$ denote the Wiener-Lévy processes [66]. These equations include the linear mean square estimation (LMSE) [65] with model parameter $C_{\phi}=8$ according to previous work [52]. The SGS mixing frequency $\Omega$ is expressed as $\Omega=$ $\left(\gamma+\gamma_{t}\right) /\left(\langle\rho\rangle \Delta^{2}\right)[49]$, where $\gamma_{t}$ denotes the SGS diffusivity. The LMSE model pa- 
rameter $C_{\phi}=8$ is set consistent with previous work [52]. The term $\epsilon_{t}$ denotes the total rate of turbulent dissipation, including both SGS and resolved contributions,

$$
\epsilon_{t}=k \Omega+\frac{1}{\langle\rho\rangle}\left\langle\tau_{i j}\right\rangle \frac{\partial\left\langle u_{i}\right\rangle_{L}}{\partial x_{j}}
$$

where $k=\tau\left(u_{i}, u_{i}\right) / 2$ denotes the SGS kinetic energy. The Fokker-Planck equation conjugate to the system of SDEs constitutes the modeled En-FDF transport equation [62]

$$
\begin{aligned}
& \frac{\partial \mathscr{F}_{e n}}{\partial t}+\frac{\partial\left(\left\langle u_{i}\right\rangle_{L} \mathscr{F}_{e n}\right)}{\partial x_{i}}=\frac{\partial}{\partial x_{j}}\left(\left(\gamma+\gamma_{t}\right) \frac{\partial\left(\mathscr{F}_{e n} /\langle\rho\rangle\right)}{\partial x_{j}}\right)+C_{\phi} \Omega \frac{\partial}{\partial \hat{\phi}_{\alpha}}\left[\left(\hat{\phi}_{\alpha}-\left\langle\phi_{\alpha}\right\rangle_{L}\right) \mathscr{F}_{\text {en }}\right] \\
& -\frac{\epsilon_{t}}{T} \frac{\partial \mathscr{F}_{e n}}{\partial \hat{s}}-\frac{1}{T} \sum_{\alpha=1}^{N_{s}} C_{\phi} \Omega\left[\mu_{\alpha}\left(\hat{\phi}_{\alpha}-\left\langle\phi_{\alpha}\right\rangle_{L}\right)\right] \frac{\partial \mathscr{F}_{e n}}{\partial \hat{s}}+\frac{1}{T} C_{\phi} \Omega\left[\left(h-\langle h\rangle_{L}\right)\right] \frac{\partial \mathscr{F}_{e n}}{\partial \hat{s}} \\
& -\sum_{\alpha=1}^{N_{s}} \frac{\partial}{\partial \hat{\phi}_{\alpha}}\left[S_{\alpha}(\hat{\boldsymbol{\phi}}) \mathscr{F}_{e n}\right]+\frac{1}{T} \sum_{\alpha=1}^{N_{s}} \mu_{\alpha} S_{\alpha}(\hat{\boldsymbol{\phi}}) \frac{\partial \mathscr{F}_{e n}}{\partial \hat{s}}
\end{aligned}
$$

The modeled filtered entropy transport equation is the first moment obtained by multiplying this equation by entropy and integrating over scalar, entropy sample space.

We obtain

$$
\begin{aligned}
& \frac{\partial\langle\rho\rangle\langle s\rangle_{L}}{\partial t}+\frac{\partial\langle\rho\rangle\left\langle u_{i}\right\rangle_{L}\langle s\rangle_{L}}{\partial x_{i}}=\frac{\partial}{\partial x_{i}}\left(\gamma \frac{\partial\langle s\rangle_{L}}{\partial x_{i}}\right)-\frac{\partial\langle\rho\rangle \tau\left(u_{i}, s\right)}{\partial x_{i}}+\langle\rho\rangle \epsilon_{t}\left\langle\frac{1}{T}\right\rangle_{L} \\
& +\langle\rho\rangle C_{\phi} \Omega\left[\sum_{\alpha=1}^{N_{s}} \tau\left(\phi_{\alpha}, \frac{g_{\alpha}}{T}\right)-\tau\left(h, \frac{1}{T}\right)\right]+\langle\rho\rangle C_{\phi} \Omega \sum_{\alpha=1}^{N_{s}} R_{\alpha} \tau\left(\phi_{\alpha}, \ln X_{\alpha}\right)-\left\langle\frac{\rho}{T} \sum_{\alpha=1}^{N_{s}} \mu_{\alpha} S_{\alpha}\right\rangle
\end{aligned}
$$

where $g_{\alpha}$ is the specific Gibbs free energy. Comparing this equation and the exact entropy transport equation (Eq. (5)) gives the closure of all entropy generation modes 
as implied by the En-FDF

$$
\begin{aligned}
\left\langle S_{g_{V}}\right\rangle & =\left\langle\frac{1}{T} \tau_{i j} \frac{\partial u_{i}}{\partial x_{j}}\right\rangle \approx\left\langle\frac{1}{T}\right\rangle_{L}\langle\rho\rangle \epsilon_{t} \\
\left\langle S_{g_{H}}\right\rangle & =\left\langle\gamma \frac{c_{p}}{T^{2}} \frac{\partial T}{\partial x_{i}} \frac{\partial T}{\partial x_{i}}\right\rangle \approx\langle\rho\rangle C_{\phi} \Omega\left[\sum_{\alpha=1}^{N_{s}} \tau\left(\phi_{\alpha}, \frac{g_{\alpha}}{T}\right)-\tau\left(h, \frac{1}{T}\right)\right] \\
\left\langle S_{g_{M}}\right\rangle & =\left\langle\sum_{\alpha=1}^{N_{s}} \gamma R_{\alpha} \frac{1}{X_{\alpha}} \frac{\partial \phi_{\alpha}}{\partial x_{i}} \frac{\partial X_{\alpha}}{\partial x_{i}}\right\rangle \approx\langle\rho\rangle C_{\phi} \Omega \sum_{\alpha=1}^{N_{s}} R_{\alpha} \tau\left(\phi_{\alpha}, \ln X_{\alpha}\right)
\end{aligned}
$$

The contribution of chemical reaction $\left\langle S_{g_{C}}\right\rangle=-\left\langle\frac{\rho}{T} \sum_{\alpha=1}^{N_{s}} \mu_{\alpha} S_{\alpha}\right\rangle$ (the last term on the RHS of Eq. (5)) is closed in the En-FDF formulation. The numerical solution of EnFDF involves a hybrid finite-difference (FD)/Lagrangian Monte Carlo (MC) method [48-51,54,55] in which an ensemble of MC particles represent the En-FDF. Each particle carries information pertaining to position, scalars and entropy. These variables are evolved by temporal integration of the SDEs. This integration is performed via the Euler-Maruyamma discretization [67]. The unclosed terms in FD solver are obtained from $\mathrm{MC}$ by ensemble averaging inside an ensemble domain centered around each grid point. Construction of the filtered values from the MC solver is done on an ensemble domain with the size equal to the filter characteristic width.

\section{Flow configuration and numerical specifications}

The flow configuration is the turbulent nonpremixed piloted methane jet flame (Sandia Flame D) [63]. The flame consists of a jet with the composition of $25 \%$ methane and $75 \%$ air by volume. The fuel nozzle is placed in a coflow of air and the flame is stabilized by a substantial pilot. The Reynolds number for the main jet is $R e=22,400$ based on the nozzle diameter $D=7.2 \mathrm{~mm}$ and the bulk jet velocity $49.6 \mathrm{~m} / \mathrm{s}$. All details regarding this flame is available on the web [68]. As the flame operates near equilibrium, the combustion chemistry is implemented using the flamelet concept [69]. In 
this implementation the detailed chemical mechanism of the Gas Research Institute (GRI 2.11) [70] is employed in a laminar, one-dimensional counterflow (opposed jet) flame. Near equilibrium, the thermo-chemical variables can be considered as functions of only mixture fraction $\xi$, which is carried as an additional passive scalar. The constant strain rate of $100 s^{-1}$ is chosen consistent with that used in previous FDF predictions of this flame $[52,59]$. The flow variables at the inflow are set similar to those in the experiment, including the inlet profiles of velocity and mixture fraction. A time-dependent forcing function consisting of oscillating axisymmetric and helical perturbations is implemented at the inlet to excite the flow [71]. The forcing amplitude is set according to the experimentally measured turbulent intensity of the streamwise velocity at the inlet. The computational domain consist of a three-dimensional (3D) Cartesian mesh with uniform grid spacings along all coordinate directions. The domain spans a region of $18 D \times 10 D \times 10 D$ in the streamwise $(x)$, and lateral $(y, z)$ directions, respectively. The number of grid points are $91 \times 101 \times 101$ in the $x, y$ and $z$ directions, respectively. Equally spaced grid points are used for FD solution of mass, momentum, scalar and entropy transport equations. The FD solver is based on a compact parameter scheme [72]. The Navier-Stokes characteristic boundary conditions [73] are used on all FD domain boundaries. The LES filter characteristic width is considered as $\Delta=2 \sqrt[3]{\Delta x \Delta y \Delta z}$ where $\Delta x, \Delta y$ and $\Delta z$ denote grid spacing in the corresponding directions. The particles are supplied at the inlet and are transported freely within the domain. In total, there are about 3.4 million MC particles within the domain at all times and there are approximately $48 \mathrm{MC}$ particles available at each grid point for ensemble averaging. Per results of extensive previous studies, $[48-51,54,55]$ this number is sufficient to yield an excellent statistical accuracy with minimal dispersion errors. 


\section{Results}

The primary objectives of these simulation are to validate the En-FDF predictions and to conduct analysis of entropy generation in a turbulent nonpremixed flame. Validation of En-FDF is demonstrated by comparing the entropy statistics with the experimental data. Extensive assessments of hydrodynamic and thermochemistry fields predicted by the FDF are shown in previous contributions [52,59]. The experimental data for entropy statistics are constructed from the instantaneous temperature and species mass fractions reported of the experiment. Entropy statistics from the En-FDF is obtained by time-averaging the filtered fields during 7 flow through times. A total of 60, 000 samples for each variable are averaged locally in this recording period. The notations $\bar{Q}$ and $R M S(Q)$ denote, respectively, the time-averaged mean and root mean square fields for a variable $Q$. The radial $r=\sqrt{y^{2}+z^{2}}$ distribution of the mean filtered entropy is shown at $x / D=7.5,15$ (Fig. 1). At both locations the En-FDF prediction of mean entropy shows favorable agreement with the data. The RMS values obtained from the En-FDF are the resolved and total components. The former is the second moment of filtered entropy, $R(s, s)^{1 / 2}$ where $R(s, s)=\overline{\langle s\rangle_{L}^{2}}-\overline{\langle s\rangle}_{L}^{2}$; the latter, $r(s, s)^{1 / 2}$, contains contribution of both resolved and SGS fields and thus corresponds with the unfiltered RMS field, $r(s, s)=(R(s, s)+\overline{\tau(s, s)})^{1 / 2} \approx\left(\overline{s^{2}}-\bar{s}^{2}\right)^{1 / 2}$ [74]. As shown in Fig. 1, the resolved and total RMS values of the entropy are also in good agreements with the data at both locations. Similar agreement is observed at other axial locations.

The instantaneous generation of entropy in various regions of the flame is depicted in Fig. 2. The entropy generation contours are overlaid on temperature iso-surfaces. The region close to the nozzle is dominated by the molecular diffusion and the flow resembles a laminar jet. Farther downstream, the growth of perturbations causes for- 
mation of large-scale coherent structures and the flow becomes fully turbulent. As evidenced in this figure, near the nozzle the main cause of entropy generation is large gradients in the inner (jet/pilot) and the outer (pilot/coflow) shear layers. As the jet develops downstream, the entropy generation shows increased spread and peak values. In turbulent regions, entropy is produced because of the dissipation associated with turbulent mixing. The average entropy generation occurring in this flow is illustrated in Fig. 3 at two locations. This figure also shows the influence of each individual irreversibility effect on the overall entropy generation. Near the inlet $(x / D=5)$ the irreversibility by heat conduction peaks in the inner shear layer. The effect of chemical reaction is equally important but it occurs in the pilot region (between the inner and outer shear layers) where the rate of reaction is high. At $x / D=15$, the effect of heat conduction becomes dominant near the centerline because of the scalar (temperature) dissipation associated with turbulent structures. Also, as turbulent jet spreads, the irreversibility by chemical reaction extends to wider radial locations. At both locations, the total entropy generation shows peak values at the intersection of heat conduction and chemical reaction profiles. It is also observed that the entropy generation by mass diffusion is less significant and that by viscous dissipation is negligible (not shown), as expected, in low Mach number flows. It is noted that experimental data for direct assessment of entropy generation predictions is not available for this flame; however, close agreement of filtered entropy with the data (Fig. 1) is an indication of accurate prediction of entropy generation terms (Eq. (5)). The dependency of entropy generation predicted by the En-FDF with respect to the model parameter $C_{\phi}$ is shown in Fig. 4. It is evidenced that the entropy generation effects show insignificant variations with this parameter in the present study. For chemical reaction this results from small influence of this parameter on the filtered quantities in this flame [56]. For mass dif- 
fusion and heat conduction, although this parameter appears explicitly in the En-FDF representation of these terms (Eq. (13)), but an increase in $C_{\phi}$ causes faster decay of the second order moments. As a results, the corresponding filtered entropy generation remains mostly insensitive to this constant. As shown, the overall entropy generation is also almost independent of $C_{\phi}$.

The En-FDF prediction of the individual entropy generation effects allows identification of dominant irreversibility mechanisms. This understanding subsequently guides the adjustment of flow conditions and geometrical parameters to minimize the irreversibilities. To demonstrate the effectiveness of this approach, we show the extent of variation of entropy generation with respect to inflow-stream turbulence intensity. We vary the inlet velocity perturbations by multiplying the amplitude of velocity perturbations in the radial direction to obtain $u^{\prime} / u_{\text {exp }}^{\prime}=1.0,1.014,1.039,1.087$; where $u^{\prime}$ and $u_{\text {exp }}^{\prime}$ are the RMS of the imposed velocity perturbation and that given by the experiment; we have $u^{\prime}={\overline{u_{i}^{\prime} u_{i}^{\prime}}}^{1 / 2}$ where $u_{i}^{\prime}$ is the RMS of velocity perturbations in each direction. Increasing the inlet velocity perturbations enhances the flapping excitation of the jet and causes generation of higher RMS values for velocity and scalar variables at downstream locations, as shown in Fig. 5. This figure illustrates the radial variation of the resolved RMS of velocity $\left(R\left(u_{i}, u_{i}\right)\right)^{1 / 2}$ and the total RMS of mixture fraction. The corresponding entropy generation is shown in Fig. 6. It is noticed that all entropy generation modes exhibit increased values with turbulent intensity. The heat conduction, mass diffusion and viscous dissipation components occur because of the dissipation associated with turbulent kinetic/scalar energy. These components become more dissipative as the turbulence intensifies, which is manifested as increase in production of entropy. The irreversibility by chemical reaction is due to high reaction rates 
and shows an increasing trend with turbulent mixing. A large increase is observed in the entropy generation by heat conduction near the centerline which is attributed to more rapid formation and advection of vortical structures, causing larger temperature variations in this region. This mode also exhibits a secondary peak at farther radial locations caused by formation of coherent structures in the outer shear layer. The contribution of each entropy generation mode to the overall availability loss is obtained by integrating over the entire computational domain. The exergy destruction contributions are listed in Table 1 for various inflow velocity RMS values. The total exergy destruction indicates the loss of availability (work-producing capacity) of the fluid exiting the domain. The last column corresponds to the second law efficiency, defined

as the ratio of available energy to exergy influx at the inlet $\eta_{I I}=\left(1-\frac{T_{0} \overline{\left\langle S_{g}\right\rangle}}{e_{\text {xin }}}\right) \times 100$, where $e_{x i n}$ is the rate of exergy influx at the inlet, $\overline{\left\langle S_{g}\right\rangle}$ is the mean filtered total entropy generation and $T_{0}=291 K$ is the dead state temperature. All entropy generation effects show increased values with higher turbulence intensity. As a result, the second law efficiency decreases significantly from $92.2 \%$ to $79.8 \%$, which indicates that the exergy of the outflowing fluid decreases due to increased irreversible losses at higher turbulence intensities.

\section{Concluding remarks}

Large eddy simulation (LES) is applied for predicting and analyzing the entropy generation in turbulent combustion. The entropy transport equation is considered in LES. This equation includes several unclosed terms due to entropy generation effects: viscous dissipation, heat transfer, mass diffusion and chemical reaction. The subgrid scale (SGS) closure is provided by the entropy filtered density function (EnFDF), which contains the complete statistical information on entropy and scalars. The 
methodology is applied to a turbulent nonpremixed jet flame (Sandia Flame D) and predictions are assessed by comparing with the experimental data. Entropy generation contributions are obtained from the En-FDF and analyzed. It is shown that heat conduction and chemical reaction are the most dominant modes of entropy production in this flame. The effectiveness of this approach to identify and to reduce irreversible losses is demonstrated by analyzing the sensitivity of entropy generation to the turbulent intensity at the inflow. Increasing this parameter is shown to increase all irreversibilities, which leads to reduced second law efficiency.

\section{References}

[1] J. H. Keenan, Availability and irreversibility in thermodynamics, Br. J. Appl. Phys. 2 (7) (1951) 183-192.

[2] G. Gouy, About available energy, J. Phys. II 8 (1889) 501-518.

[3] A. Stodola, Steam and Gas Turbines, McGraw-Hill, New York, NY, 1910.

[4] A. Bejan, Fundamentals of exergy analysis, entropy generation minimization, and the generation of flow architecture, Int. J. Energy Res. 26 (7) (2002) 545-565.

[5] A. Bejan, Entropy generation minimization: The new thermodynamics of finite-size devices and finite-time processes, J. Appl. Phys. 79 (3) (1996) 1191-1218.

[6] V. A. Mironova, A. M. Tsirlin, V. A. Kazakov, R. S. Berry, Finite-time thermodynamics: Exergy and optimization of time-constrained processes, J. Appl. Phys. 76 (2) (1994) 629_ 636.

[7] B. Andresen, Current trends in finite-time thermodynamics, Angew. Chem. Int. Ed. 50 (12) (2011) 2690-2704. 
[8] S. K. Som, A. Datta, Thermodynamic irreversibilities and exergy balance in combustion processes, Prog. Energy Combust. Sci. 34 (3) (2008) 351-376.

[9] N. Lior, W. Sarmiento-Darkin, H. S. Al-Sharqawi, The exergy fields in transport processes: Their calculation and use, Energy 31 (5) (2006) 553-578.

[10] C. D. Rakopoulos, C. N. Michos, Generation of combustion irreversibilities in a spark ignition engine under biogas-hydrogen mixtures fueling, Int. J. Hydrogen Energy 34 (10) (2009) 4422-4437.

[11] S. Ugarte, M. Metghalchi, Evolution of adiabatic availability and its depletion through irreversible processes, Int. J. of Exergy 2 (2) (2005) 109-119.

[12] J. F. Klausner, Y. Li, M. Darwish, R. Mei, Innovative diffusion driven desalination process, J. Energy Res. Technol. 126 (2004) 219-225.

[13] U. Narusawa, The second-law analysis of convective pattern change in a rectangular cavity, J. Fluid Mech. 392 (1999) 361-377.

[14] Z. W. Li, S. K. Chou, C. Shu, W. M. Yang, Entropy generation during microcombustion, J. Appl. Phys. 97 (8) (2005) 084914-1-8.

[15] P. Rezac, H. Metghalchi, A brief note on the historical evolution and present state of exergy analysis, Int. J. Exergy 1 (4) (2004) 426-437.

[16] B. S. Yilbas, Entropy production during laser picosecond heating of copper, J. Energy Res. Technol. 124 (2002) 204-213.

[17] E. P. Gyftopoulos, G. P. Beretta, Entropy generation rate in a chemically reacting system, J. Energy Res. Technol. 115 (1993) 208-212.

[18] A. Datta, S. Som, Energy and exergy balance in a gas turbine combustor, Proc. Inst. Mech. Eng. Part A J. Power Energy 213 (1) (1999) 23-32. 
[19] T. E. Hutchins, M. Metghalchi, Energy and exergy analyses of the pulse detonation engine, J. Eng. Gas Turb. Power 125 (4) (2003) 1075-1080.

[20] N. M. Jubeh, Exergy analysis and second law efficiency of a regenerative brayton cycle with isothermal heat addition, Entropy 7 (3) (2005) 172-187.

[21] P. Chavannavar, J. Caton, Destruction of availability (exergy) due to combustion processes: a parametric study, P. I. Mech. Eng. A-J. Pow. 220 (7) (2006) 655-668.

[22] I. Sezer, I. Altin, A. Bilgin, Exergetic analysis of using oxygenated fuels in spark-ignition (SI) engines, Energy Fuels 23 (4) (2009) 1801-1807.

[23] S. Chen, J. Li, H. Han, Z. Liu, C. Zheng, Effects of hydrogen addition on entropy generation in ultra-lean counter-flow methane-air premixed combustion, Int. J. Hydrogen Energy 35 (8) (2010) 3891-3902.

[24] H. Teng, C. M. Kinoshita, S. M. Masutani, J. Zhou, Entropy generation in multicomponent reacting flows, J. Energy Res. Technol. 120 (3) (1998) 226-232.

[25] A. Datta, Entropy generation in a confined laminar diffusion flame, Combust. Sci. Technol. 159 (1) (2000) 39-56.

[26] K. Nishida, T. Takagi, S. Kinoshita, Analysis of entropy generation and exergy loss during combustion, Proc. Combust. Inst. 29 (1) (2002) 869-874.

[27] A. Datta, Effects of gravity on structure and entropy generation of confined laminar diffusion flames, Int. J. Therm. Sci. 44 (5) (2005) 429-440.

[28] S. Z. Shuja, B. S. Yilbas, M. Khan, Entropy generation in laminar jet: Effect of velocity profiles at nozzle exit, Heat Mass Transfer 42 (9) (2006) 771-777.

[29] A. M. Briones, A. Mukhopadhyay, S. K. Aggarwal, Analysis of entropy generation in hydrogen-enriched methane-air propagating triple flames, Int. J. Hydrogen Energy 34 (2) (2009) 1074-1083. 
[30] A. Sciacovelli, V. Verda, Entropy generation minimization in a tubular solid oxide fuel cell, J. Energy Res. Technol. 132 (2010) 012601-1-11.

[31] D. Jiang, W. Yang, K. J. Chua, J. Ouyang, J. H. Teng, Analysis of entropy generation distribution in micro-combustors with baffles, Int. J. Hydrogen Energy 39 (15) (2014) $8118-8125$.

[32] U. Rana, S. Chakraborty, S. K. Som, Thermodynamics of premixed combustion in a heat recirculating micro combustor, Energy 68 (2014) 510-518.

[33] N. Okong'o, J. Bellan, Entropy production of emerging turbulent scales in a temporal supercritical n-heptane/nitrogen three dimensional mixing layer, Proc. Combust. Inst. 28 (1) (2000) 467-504.

[34] N. Okong'o, J. Bellan, Direct numerical simulation of a transitional supercritical binary mixing layer: Heptane and nitrogen, J. Fluid Mech. 464 (2002) 1-34.

[35] N. A. Okong'o, J. Bellan, Small-scale dissipation in binary-species, thermodynamically supercritical, transitional mixing layers, Comput. Fluids 39 (7) (2010) 1112-1124.

[36] D. M. McEligot, K. P. Nolan, E. J. Walsh, E. Laurien, Effects of pressure gradients on entropy generation in the viscous layers of turbulent wall flows, Int. J. Heat Mass Transfer $51(5)(2008)$ 1104-1114.

[37] R. Farran, N. Chakraborty, A direct numerical simulation-based analysis of entropy generation in turbulent premixed flames, Entropy 15 (5) (2013) 1540-1566.

[38] D. Stanciu, D. Isvoranu, M. Marinescu, Y. Gogus, Second law analysis of diffusion flames, Int. J. Applied Thermodynamics 4 (1) (2001) 1-18.

[39] S. Z. Shuja, B. S. Yilbas, M. O. Budair, Local entropy generation in an impinging jet: Minimum entropy concept evaluating various turbulence models, Comput. Meth. Appl. Mech. Eng. 190 (28) (2001) 3623-3644. 
[40] O. B. Adeyinka, G. F. Naterer, Modeling of entropy production in turbulent flows, J. Fluids Eng. 126 (2004) 893-899.

[41] F. Kock, H. Herwig, Local entropy production in turbulent shear flows: A high-Reynolds number model with wall functions, Int. J. Heat Mass Transfer 47 (10-11) (2004) 22052215.

[42] H. Yapıcı, N. Kayataş, B. Albayrak, G. Baştürk, Numerical calculation of local entropy generation in a methane-air burner, Energ. Convers. Manag. 46 (11-12) (2005) 18851919.

[43] H. Herwig, F. Kock, Local entropy production in turbulent shear flows: A tool for evaluating heat transfer performance, J. Therm. Sci. 15 (2) (2006) 159-167.

[44] D. Stanciu, M. Marinescu, A. Dobrovicescu, The influence of swirl angle on the irreversibilities in turbulent diffusion flames, Int. J. Thermodyn. 10 (4) (2007) 143-153.

[45] A. Emadi, M. D. Emami, Analysis of entropy generation in a hydrogen-enriched turbulent non-premixed flame, Int. J. Hydrogen Energy 38 (14) (2013) 5961-5973.

[46] P. Givi, Filtered density function for subgrid scale modeling of turbulent combustion, AIAA J. 44 (1) (2006) 16-23.

[47] N. Ansari, F. A. Jaberi, M. R. H. Sheikhi, P. Givi, Filtered density function as a modern CFD tool, in: R. S. Maher (Ed.), Engineering Applications of CFD, Vol. 1 of Fluid Mechanics and Its Applications, International Energy and Environment Foundation, AlNajaf, Iraq, 2011, Ch. 1, pp. 1-22.

[48] P. J. Colucci, F. A. Jaberi, P. Givi, S. B. Pope, Filtered density function for large eddy simulation of turbulent reacting flows, Phys. Fluids 10 (2) (1998) 499-515.

[49] F. A. Jaberi, P. J. Colucci, S. James, P. Givi, S. B. Pope, Filtered mass density function for large eddy simulation of turbulent reacting flows, J. Fluid Mech. 401 (1999) 85-121. 
[50] L. Y. M. Gicquel, P. Givi, F. A. Jaberi, S. B. Pope, Velocity filtered density function for large eddy simulation of turbulent flows, Phys. Fluids 14 (3) (2002) 1196-1213.

[51] M. R. H. Sheikhi, T. G. Drozda, P. Givi, S. B. Pope, Velocity-scalar filtered density function for large eddy simulation of turbulent flows, Phys. Fluids 15 (8) (2003) 23212337.

[52] M. R. H. Sheikhi, T. G. Drozda, P. Givi, F. A. Jaberi, S. B. Pope, Large eddy simulation of a turbulent nonpremixed piloted methane jet flame (Sandia Flame D), Proc. Combust. Inst. 30 (2005) 549-556.

[53] T. G. Drozda, M. R. H. Sheikhi, C. K. Madnia, P. Givi, Developments in formulation and application of the filtered density function, Flow Turbul. Combust. 78 (2007) 35-67.

[54] M. R. H. Sheikhi, P. Givi, S. B. Pope, Velocity-scalar filtered mass density function for large eddy simulation of turbulent reacting flows, Phys. Fluids 19 (9) (2007) 095106 $1-21$.

[55] M. R. H. Sheikhi, P. Givi, S. B. Pope, Frequency-velocity-scalar filtered mass density function for large eddy simulation of turbulent flows, Phys. Fluids 21 (7) (2009) 075102 1-14.

[56] M. Yaldizli, K. Mehravaran, F. A. Jaberi, Large-eddy simulations of turbulent methane jet flames with filtered mass density function, Int. J. Heat Mass Transfer 53 (11-12) (2010) 2551-2562.

[57] S. L. Yilmaz, M. B. Nik, M. R. H. Sheikhi, P. A. Strakey, P. Givi, An irregularly portioned lagrangian monte carlo method for turbulent flow simulation, J. Sci. Comput. 47 (1) (2011) 109-125.

[58] M. Nik, S. Yilmaz, M. R. H. Sheikhi, P. Givi, Grid resolution effects on VSFMDF/LES, Flow Turbul. Combust. 85 (3-4) (2010) 677-688. 
[59] M. B. Nik, S. L. Yilmaz, P. Givi, M. R. H. Sheikhi, S. B. Pope, Simulation of Sandia flame D using velocity-scalar filtered density function, AIAA J. 48 (7) (2010) 1513-1522.

[60] N. Ansari, P. H. Pisciuneri, P. A. Strakey, P. Givi, Scalar-filtered mass-density-function simulation of swirling reacting flows on unstructured grids, AIAA J. 50 (11) (2012) $2476-2482$.

[61] M. R. H. Sheikhi, M. Safari, H. Metghalchi, Large eddy simulation for local entropy generation analysis of turbulent flows, J. Energy Res. Technol. 134 (4) (2012) 041603 $1-6$.

[62] M. Safari, M. R. H. Sheikhi, Large eddy simulation for prediction of entropy generation in a nonpremixed turbulent jet flame, J. Energy Res. Technol. 136 (2) (2014) 022002 1-6.

[63] J. H. Frank, R. S. Barlow, Simultaneous Rayleigh, Raman, and LIF measurements in turbulent premixed methane-air flames, Proc. Combust. Inst. 27 (1998) 759-766.

[64] M. Safari, M. R. H. Sheikhi, M. Janbozorgi, H. Metghalchi, Entropy transport equation in large eddy simulation for exergy analysis of turbulent combustion systems, Entropy 12 (3) (2010) 434-444.

[65] S. B. Pope, Turbulent Flows, Cambridge University Press, Cambridge, UK, 2000.

[66] I. I. Gikhman, A. V. Skorokhod, Stochastic Differential Equations, Springer-Verlag, New York, NY, 1972.

[67] P. E. Kloeden, E. Platen, H. Schurz, Numerical Solution of Stochastic Differential Equations through Computer Experiments, corrected second printing Edition, SpringerVerlag, New York, NY, 1997.

[68] R. S. Barlow, Sandia National Laboratories, TNF Workshop website, http://www . ca. sandia.gov/TNF (2011). 
[69] N. Peters, Turbulent Combustion, Cambridge University Press, Cambridge, UK, 2000.

[70] C. T. Bowman, R. K. Hanson, W. C. Gardiner, V. Lissianski, M. Frenklach, M. Goldenberg, G. P. Smith, D. R. Crosley, D. M. Golden, GRI-mech 2.11-an optimized detailed chemical reaction mechanism for methane combustion and NO formation and reburning, Report GRI-97/0020, Gas Research Institute, Chicago, IL (1997).

[71] I. Danaila, B. J. Boersma, Direct numerical simulation of bifurcating jets, Phys. Fluids 12 (5) (2000) 1255-1257.

[72] C. A. Kennedy, M. H. Carpenter, Several new numerical methods for compressible shearlayer simulations, Appl. Num. Math. 14 (1994) 397-433.

[73] T. J. Poinsot, S. K. Lele, Boundary conditions for direct simulations of compressible viscous flows, J. Comput. Phys. 101 (1992) 104-129.

[74] B. Vreman, B. Geurts, H. Kuerten, Large-eddy simulation of the turbulent mixing layer, J. Fluid Mech. 339 (1997) 357-390. 


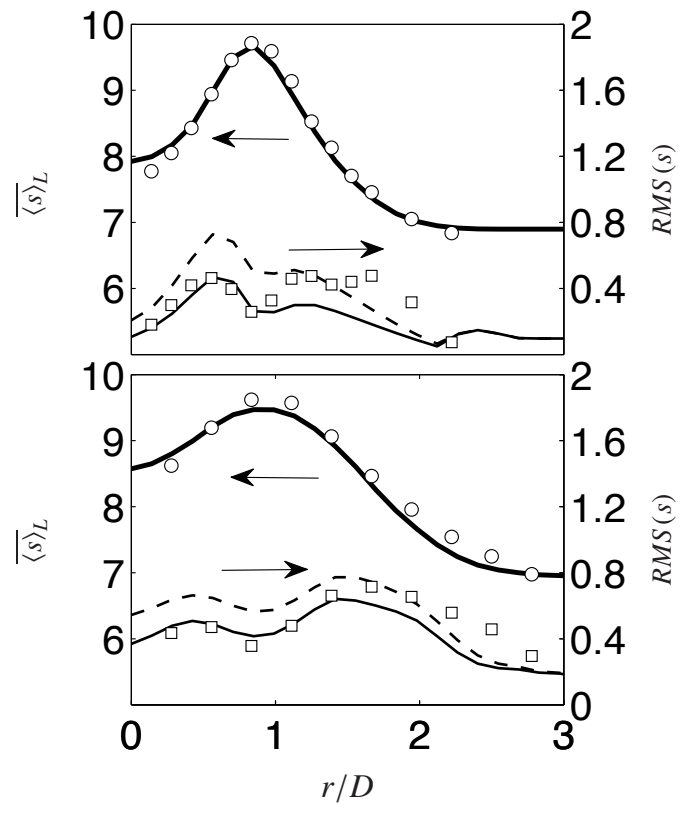

Fig. 1. Radial variation of mean and RMS entropy $[\mathrm{kJ} / \mathrm{kg} . \mathrm{K}]$ at $x / D=7.5$ (top) and $x / D=15$ (bottom). The thin solid and dashed lines denote the resolved and total RMS values, respectively. Symbols denote the experimental data. 


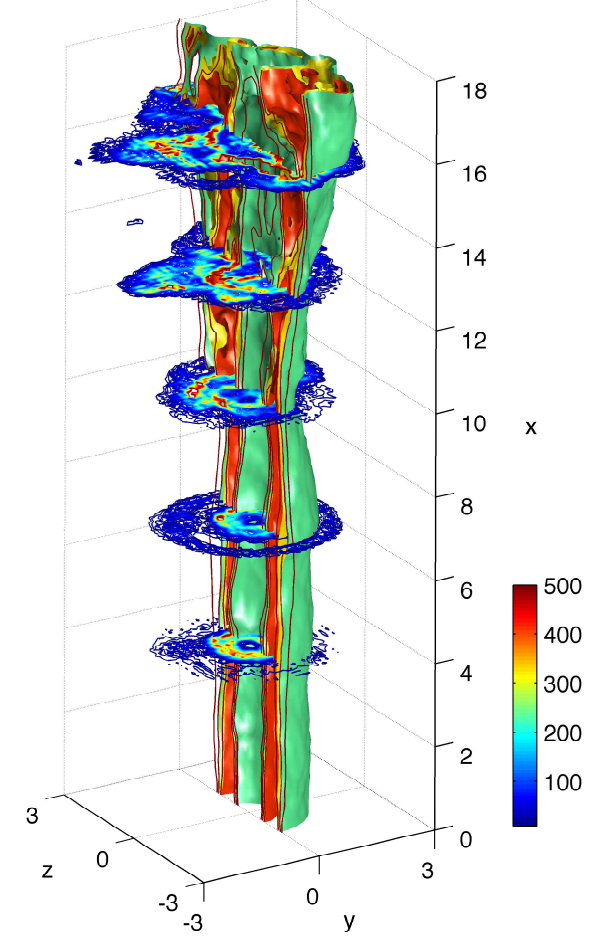

Fig. 2. Instantaneous iso-surfaces of temperature (red: $1700 \mathrm{~K}$; yellow: $1400 \mathrm{~K}$; green: $1100 \mathrm{~K}$ ) superimposed by contours of entropy generation $\left[k J / K . m^{3} . s\right]$ at different axial locations. 


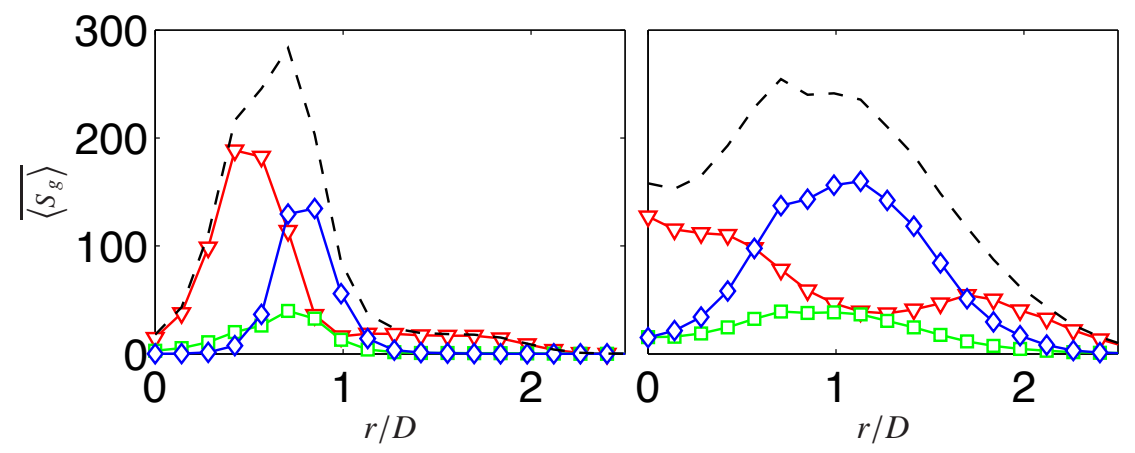

Fig. 3. Radial variation of mean entropy generation $\left[k J / K . m^{3} . s\right]$ by heat conduction $(\nabla)$, mass diffusion $(\square)$, chemical reaction $(\diamond)$ as well as the total entropy generation $(--)$ at $x / D=5$ (left) and $x / D=15$ (right).

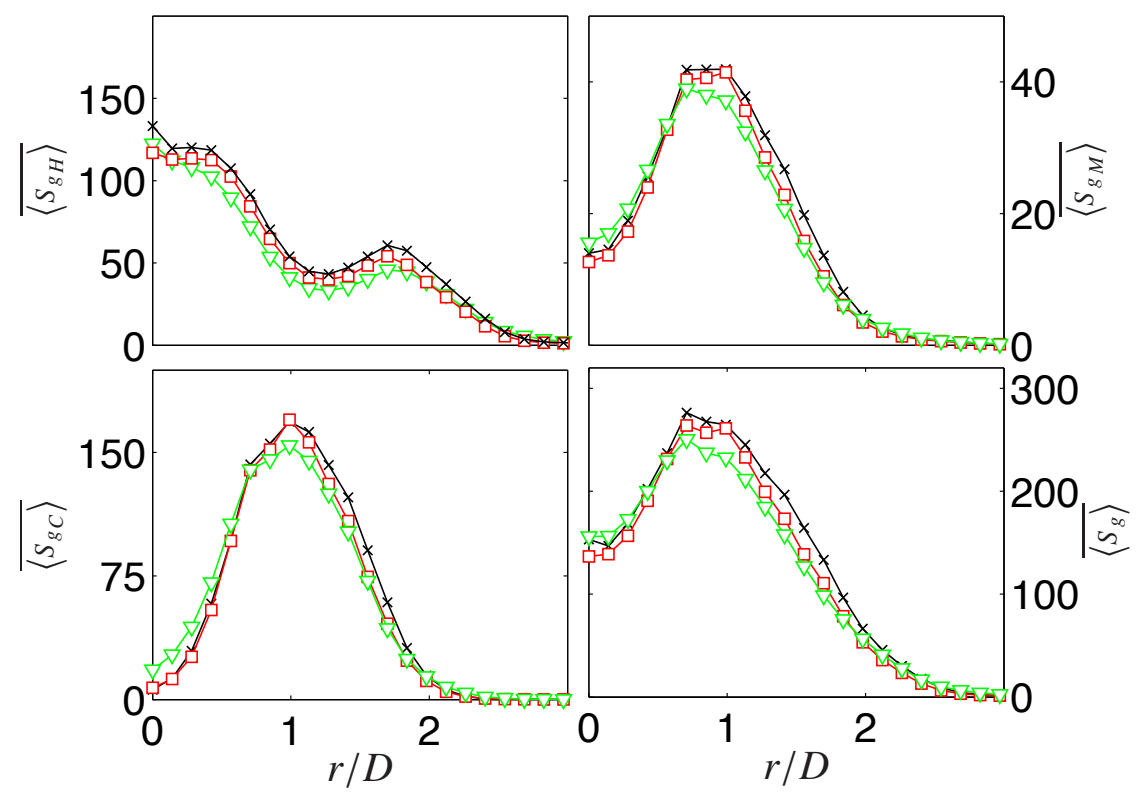

Fig. 4. Variation of entropy generation effects $\left[k J / K . m^{3} . s\right]$ with respect to model constant $C_{\phi}$ at $x / D=15$. Symbols correspond to $C_{\phi}=6(\nabla) ; C_{\phi}=10(\square)$; and $C_{\phi}=15(\times)$. 


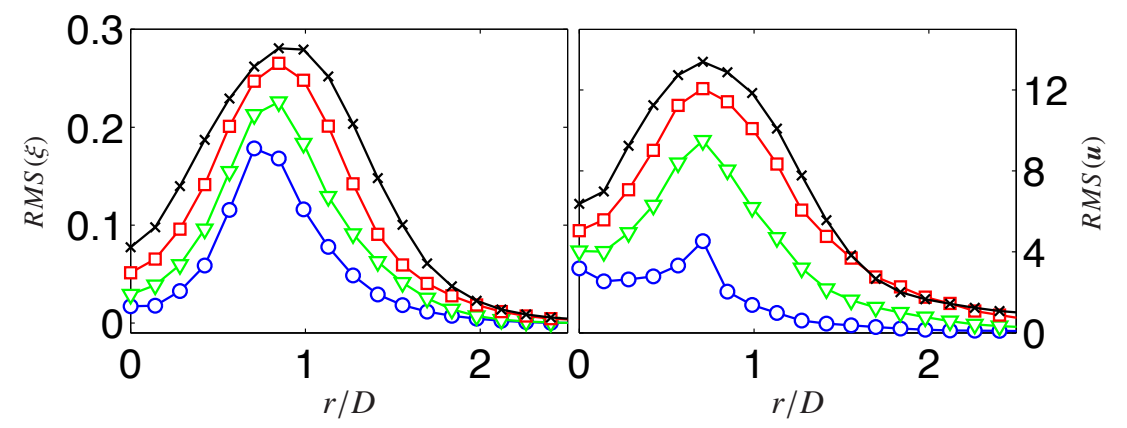

Fig. 5. Radial variation of total RMS of mixture fraction (left) and resolved RMS of velocity $[m / s]$ (right) at $x / D=10$. Symbols correspond to inflow stream velocity RMS, $u^{\prime} / u_{\exp }^{\prime}=1.0$ (○), $1.014(\nabla), 1.039(\square)$ and $1.087(\times)$.

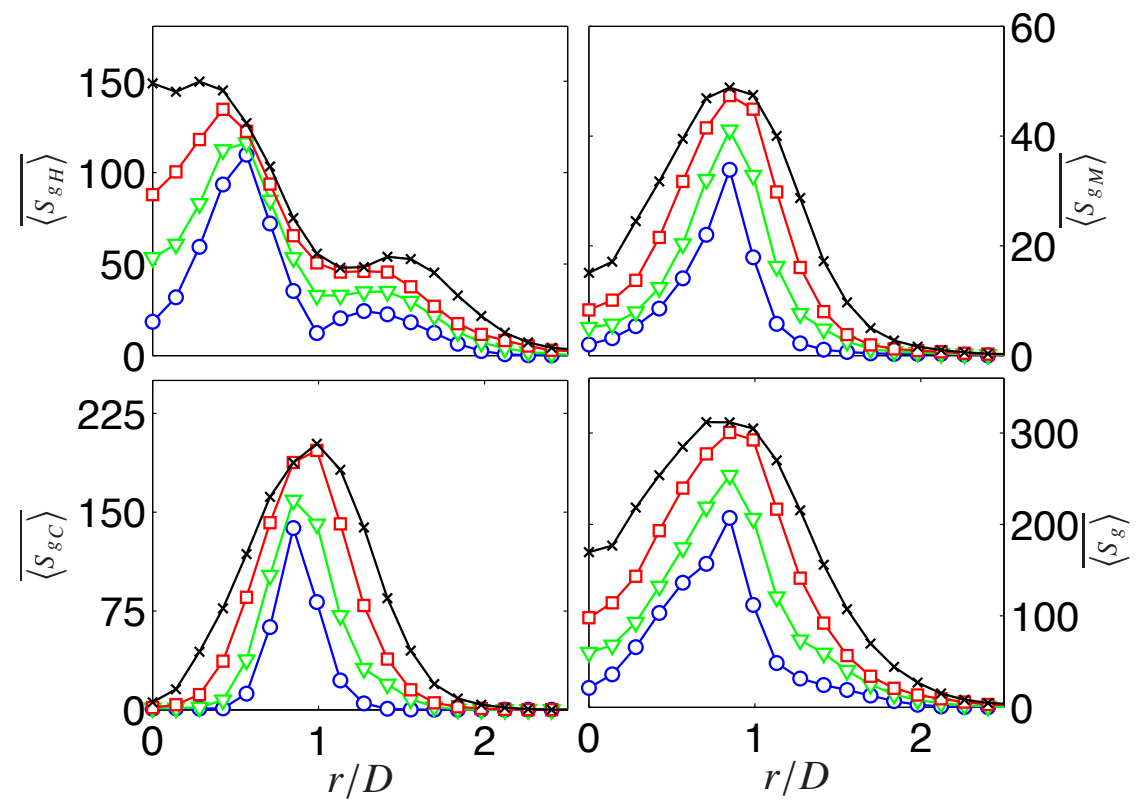

Fig. 6. Variation of entropy generation effects $\left[k J / K . \mathrm{m}^{3} . \mathrm{s}\right]$ with respect to turbulence intensity at $x / D=10$. Symbols correspond to inflow stream velocity RMS, $U^{\prime} / u_{\text {exp }}^{\prime}=1.0(0), 1.014$ $(\nabla), 1.039(\square)$ and $1.087(\times)$. 
Table 1: Effect of turbulence intensity on exergy loss $\left(\mathrm{J} / \mathrm{m}^{3} . \mathrm{s}\right)$.

\begin{tabular}{ccccccc}
\hline$u^{\prime} / u_{\text {exp }}^{\prime}$ & $T_{0} \overline{\left\langle S_{g_{H}}\right\rangle}$ & $T_{0} \overline{\left\langle S_{g_{M}}\right\rangle}$ & $T_{0} \overline{\left\langle S_{g_{V}}\right\rangle}$ & $T_{0} \overline{\left\langle S_{g_{C}}\right\rangle}$ & $T_{0} \overline{\left\langle S_{g}\right\rangle}$ & $\eta_{I I}(\%)$ \\
\hline \hline 1.0 & 762 & 172 & 0.0314 & 509 & 1440 & 92.2 \\
1.014 & 1170 & 317 & 0.0616 & 1090 & 2580 & 86.0 \\
1.039 & 1300 & 377 & 0.0579 & 1360 & 3030 & 83.6 \\
1.087 & 1540 & 468 & 0.0815 & 1740 & 3750 & 79.8 \\
\hline
\end{tabular}

